\title{
Weighing the vacuum with the Archimedes experiment
}

\section{S. Avino ${ }^{a, b}$, A. Basti ${ }^{c}$, E. Calloni ${ }^{d, b}$, S. Caprara ${ }^{e, f}$, M. De Laurentis ${ }^{* d, b}$, R. De Rosa ${ }^{d, b}$, G. Esposito ${ }^{b}$, F. Frasconi ${ }^{c}$, G. Gagliardi ${ }^{a, 2}$, M. Grillie ${ }^{e}$, , E. Majorana ${ }^{f}$, G. P. Pepe ${ }^{d, b}$, S. Petrarca $^{e, f}$, P. Puppo ${ }^{f}$, P. Rapagnani ${ }^{e, f}$, F. Ricci ${ }^{e, f}$, L. Rosa ${ }^{d, b}$, C. Rovelli ${ }^{g, h}$, P. Ruggi ${ }^{i}$, N. L. Saini ${ }^{e, f}$, C. Stornaiolo ${ }^{b}$, D. Stornaiuolo ${ }^{d, b}$, F. Tafuri ${ }^{d, b}$}

a INO-CNR, Comprensorio Olivetti, Via Campi Flegrei 34-80078 Pozzuoli (NA), Italy

${ }^{b}$ INFN Sezione di Napoli, Via Cintia Edificio 6, I-80126 Naples, Italy

${ }^{c}$ INFN sezione Pisa, Largo Pontecorvo 1, I-56127 Pisa, Italy

${ }^{d}$ Università Federico II Napoli, via Cinthia, I-80126, Naples, Italy

e Università di Roma La Sapienza, Piazzale A. Moro, I-00185 Roma, Italy

${ }^{f}$ INFN Sezione di Roma, Piazzale A. Moro, I-00185 Roma, Italy

${ }^{g}$ Aix Marseille Université CNRS, CPT, UMR 7332, 13288 Marseille, France

${ }^{h}$ Universitè de Toulon, CNRS, CPT, UMR 7332, 83957 La Garde, France

${ }^{i}$ European Gravitational Observatory (EGO), I-56021 Cascina, Pisa, Italy

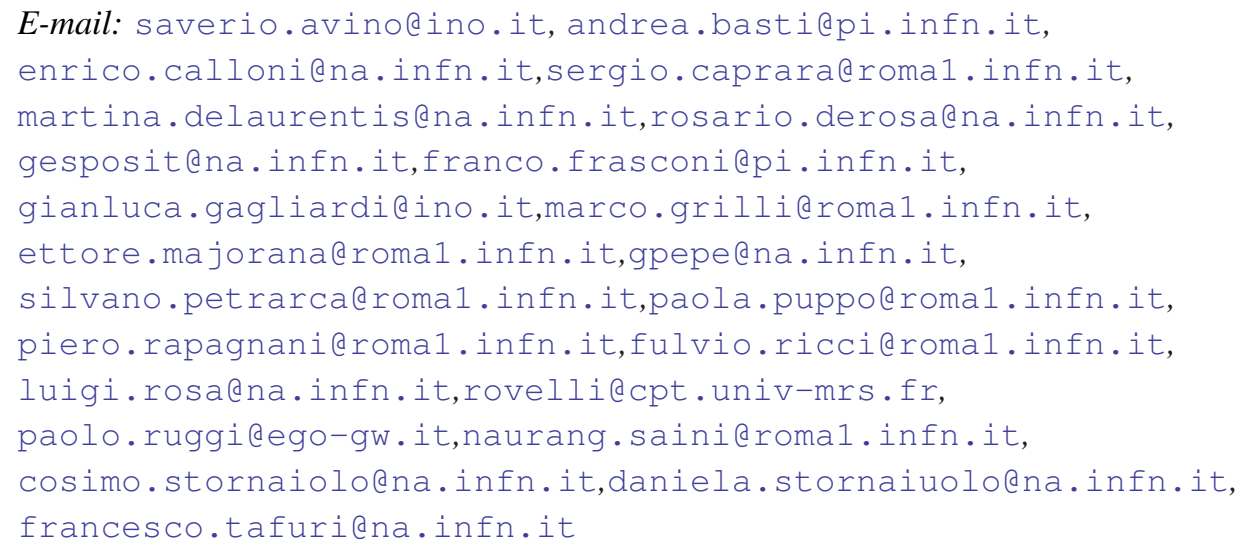

We present the status of the art of the Archimedes experiment, devoted to measure the weight of zero point energy by weighing the condensation energy of a layered type II superconductor. The experimental scheme of the Archimedes experiment is presented and the sensitivity needed for the final measurement illustrated. The results of the balance prototype are presented showing the torque sensitivity of about $10^{-11} \mathrm{Nm} / \sqrt{\mathrm{Hz}}$ in the region of frequency from about $50 \mathrm{mHz}$ up two $150 \mathrm{mHz}$. The major upgrades needed to reach the desired final sensitivity are discussed.

XII Multifrequency Behaviour of High Energy Cosmic Sources Workshop 12-17 June, 2017

Palermo, Italy

${ }^{*}$ Speaker. 


\section{Introduction}

One of the oldest questions concerning quantum mechanics and gravitation is whether vacuum fluctuations do gravitate. The question of the weight of vacuum was raised soon after the birth of quantum mechanics [1] and many theoretical attempts were performed to explore the motivations and consequences of assuming or discarging such hyphothesis [2,3]. From an experimental point of view it is remarkable that up to now not a direct experimental measurement has been carried out. Considering that nowadays the scientific community regards the Casimir effect as a macroscopic manifestation of vacuum fluctuations [4, 5], we recently proposed an experiment, named Archimedes, to test the interaction of vacuum fluctuations with gravity by weighing a suitable rigid Casimir cavity.

In various papers we have estimated the effect of the gravitation field on such a cavity on the earth (the cavity is intended as rigid)under the hypothesis that vacuum energy does interact with gravitational field and follows the strong equivalence principle: it is found that the gravitation field exerts a force on the cavity equal to the weight of the vacuum modes expelled by the cavity, in partial analogy with the Archimedes force in the fluid. The analogy is partial because the cavity follows the equivalence principle and falls with the gravitational acceleration [7].

Further, considering the present small force detectors we also provided an experimental scheme to measure the Archimedes force of vacuum: the cavity is actually a multi-layer cavity obtained using a layered superconductor of type II, like YBCO or BSCOO. In these kind of superconductors the superconducting planes are separated by dielectric planes, thus forming a natural multi-layer Casimir cavity $[8,9]$. Thus the actual measure will be the weight of the condensation energy of a layered-superconductor. The order of magnitude of the expected force to be measured is about $10^{-16} \mathrm{~N}$ at a modulation frequency of several $\mathrm{mHz}$, obtained by inducing a transition from normal to superconducting state by temperature modulation [7]. The measurement of such a tiny force requires an extremely sensitive balance, working at the temperature of about $100 \mathrm{~K}$, suspending two almost equivalent blocks of superconductor material which undergo modulate transition in thermal equilibrium with surrounding temperature modulated vessel, like in fig 1. A prototype of such a balance, working at room temperature, has been realized to test eventual show-stopper or major problems. In this paper we set the main experimental parameters, we show the relevant experimental prototype results and discuss them with respect to the final measurement.

\section{Measurement principles}

In varius papers $[7,10,11]$ it has been calculated the force exerted by the gravitational field on a rigid Casimir cavity at rest on earth: it is directed upward and it is equal:

$$
F=\frac{E_{c}}{c^{2}} \vec{g}
$$

where $\mathrm{c}$ is the speed of light and $\vec{g}$ is the earth gravitation acceleration. In the calculation of this force it is assumed that also the pressure of the vacuum fluctuation does follow the equivalence principle and thus the measurement is actually also a zero measurement of the read-shifting of vacuum pressure in gravitational field $[12,7]$. The force is extremely tiny and thus measurement must be performed by modulating the effect. 
The method proposed to modulate the Casimir energy in a rigid cavity is by modulating the reflectivity of the plates by a superconducting transition: when the plates are superconducting they are more reflective and the vacuum is better expelled from the cavity, resulting in a lowering of the weight $[13,14,15,16]$. The superconductor will be actually a layered type II superconductor, like YBCO or BiSCCO: indeed a type II superconductor is formed by planes that perform the transition to superconductivity alternate to layers that remain dielectric. Thus a layered superconductor is a natural stack of partially-reflecting, coupled Casimir cavities, whose condensation energy is expected to be influenced by vacuum fluctuation $[7,8,9]$. The percentage of condensation energy that is due to Casimir energy is still under evaluation and in the optimistic case is almost the totality [8]. The actual weight measurement will have the accuracy of the order of percent, so it will be possible to uncertain the interaction of gravitational field with vacuum fluctuations even if the contribution will prove to be of this order of magnitude.

The proposed scheme of the measurement is reported in fig 1: two discs of layered superconductor are suspended to the arm of the balance. The two discs are made of the same material but with different doping. The arm is in aluminum to maintain low weight and momentum of inertia. The temperature modulation is the same in the two discs but only one performs the superconductive transition: in this way the common phonon energy variation cancels (does not give a signal on the balance).

The discs are $300 \mathrm{~mm}$ diameter and $1 \mathrm{~mm}$ thick. They are enclosed in two metallic enclosures that are modulated in temperature and the discs temperature modulation is about $1 \mathrm{~K}$. The armlength is $1.40 \mathrm{~m}$, the mass $1.7 \mathrm{~kg}$, the total momentum of inertia is $0.72 \mathrm{~kg} \mathrm{~m}^{2}$. The modulation frequency is several $\mathrm{mHz}$, in agreement with the time needed to the samples to reach the temperature of equilibrium. The read-out optical system is a Mach-Zender interferometer illuminated with $5 \mathrm{~mW}$ laser power. The whole system is enclosed in vacuum at the transition temperature of the samples, about $100 \mathrm{~K}$.

The expected signal and sensitivity are reported in fig 4, for 1 months integration time. The sensitivity is calculated for a seismic noise equal to the measured in the Sardinia site of SOS-Enattos [17] (see discussion in last section) and the thermal noise is calculated in the hypothesis of resonance frequency of $5 \mathrm{mHz}$ and loss angle $\phi=10^{-5}$. Seismic and suspension thermal noise are expected to limit the sensitivity. Other fundamental sources of noise, like radiation pressure noise, shot noise, internal thermal noise, are negligible.

\section{The prototype}

The main aim of the prototype has been the test of the feasibility of a balance with low moment of inertia yet maintaining a low resonance frequency, a good sensitivity and capability of control in the short and long term. A picture of he prototype is reported in fig 2. The first important test has regarded the suspension joint. The balance arm is coupled to ground by soft joints whose restoring force is tuned to have a resonance frequency of about $3 \mathrm{mHz}$ when the center of mass of the arm is lying on the rotation axis. The use of joints is similar to the solution proposed in ref [18] for the realization of an absolute rotation sensor. The main difference among the balance and the rotation 


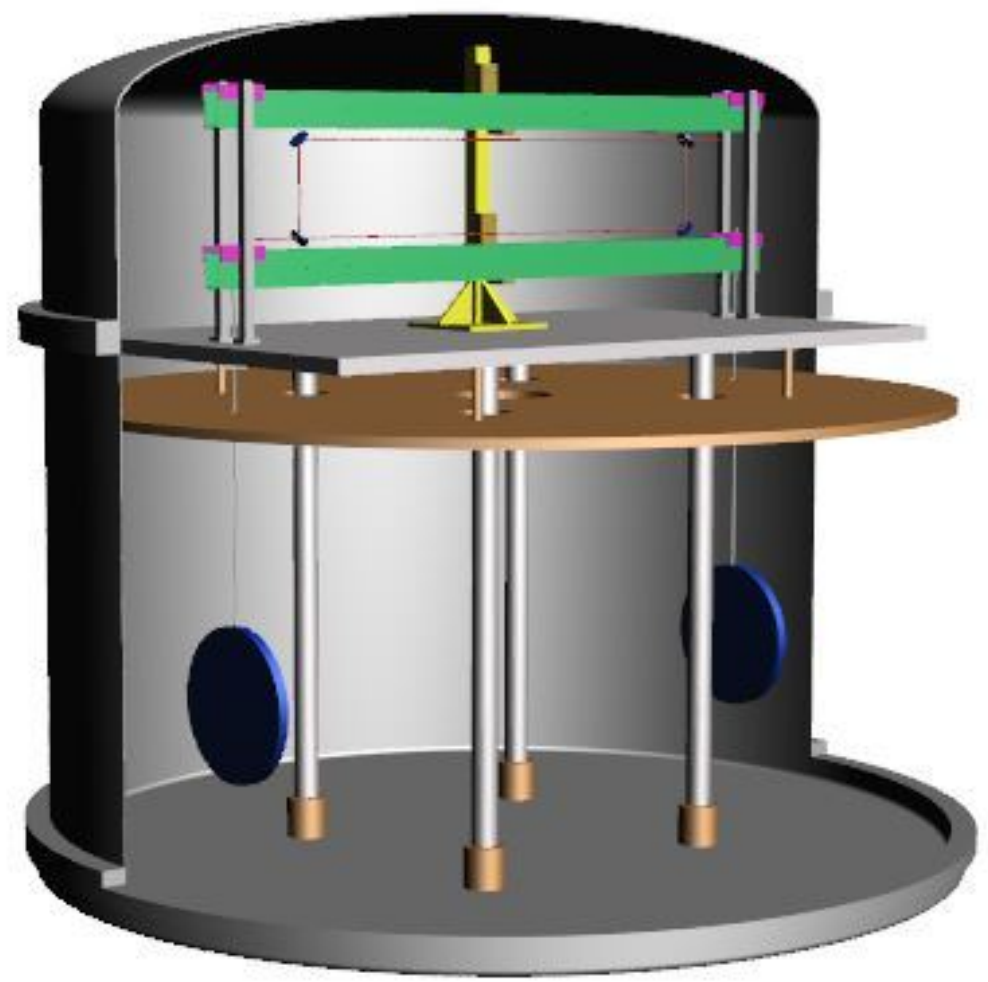

Figure 1: Scheme of the final balance. Each disc is suspended to one end-arm and surrounded by a metallic enclosure for thermal actuation (not shown). The optical read-out is a Mack-Zender interferometer and the signal is taken with respect to a reference arm.

sensor is given by optimal moment of inertia of the arm. Differently from the rotation sensor in the balance the arm should be as light as possible, compatible with the other constructive requirements, to furnish a detectable signal even when driven by the tiny vacuum force.

To recover a low resonance frequency the joints should in principle made thinner but this choice would require thickness only of a few micron, extremely difficult to machine and to work with.

This problem has been solved by designing joints, named semi-ribbon, in which the bending points separates but remain in a couple of attenuation distance, so to warrant the desired recovering stiffness.

The joint used, reported in fig 3 are made of berillium-copper. The length of the ribbon is $6 \mathrm{~mm}$, the width is $10 \mathrm{~mm}$ and the thickness is $50 \mu \mathrm{m}$. This design is optimized for the final balance, which will have the length of $1.4 \mathrm{~m}$ and the total moment of inertia (included the suspended superconducting samples) of $0.72 \mathrm{~kg} \mathrm{~m}^{2}$, but have been tested on the balance prototype to prove robustness and performances.

The balance prototype has arm-length of $0.56 \mathrm{~m}$, the arm is made of Aluminum, the moment of inertia is $0.042 \mathrm{~kg} \mathrm{~m}^{2}$. The read out is a very sensitive optical lever: the light of a SLED (Superluminescent Light-Emitting Diode) is sent to a mirror lying at the center of the upper surface 


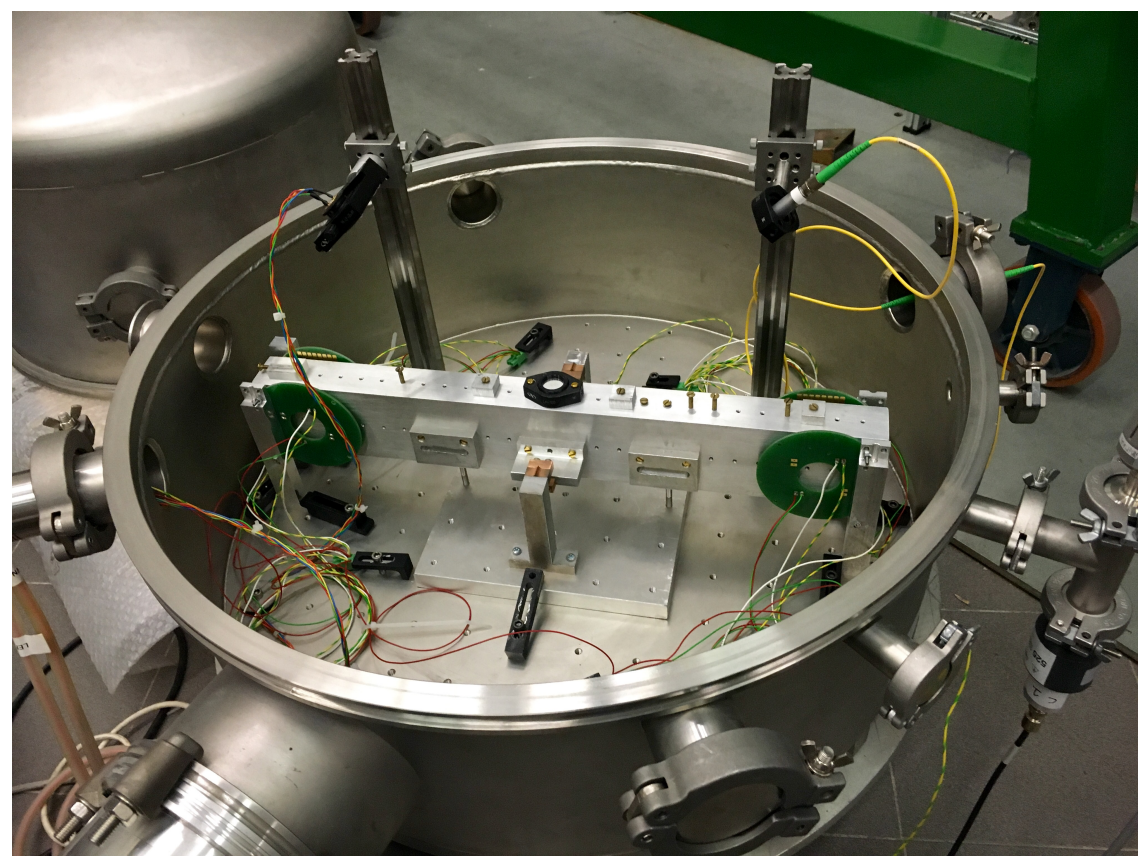

Figure 2: Picture of the prototype.

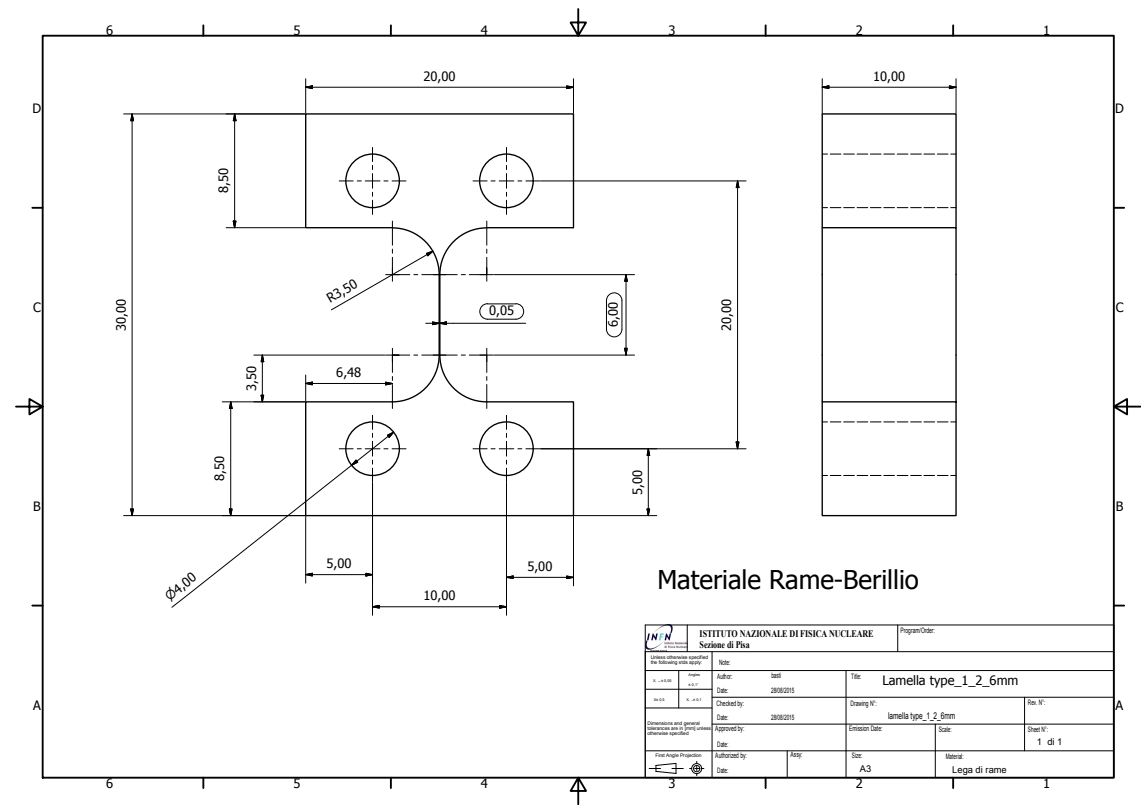

Figure 3: Design of the semi-ribbon. This configuration of joints is designed to maintain a low resonance frequency for low momentum of inertia. 
of arm. The reflected light is read by a quadrant photodiode fixed on the ground: the distance $\mathrm{d}$ form the mirror to the photodiode is $d=0.32 \mathrm{~m}$. The angle of the incident beam on the mirror is $0.05 \mathrm{rad}$. The gap among the quadrants of the photodiode is $100 \mu \mathrm{m}$ and the width SLED beam is collimated to $400 \mu \mathrm{m}$, to have a suitably high sensitivity.

The system is under vacuum, with residual pressure of about $2 \times 10^{-5}$ mbar. The balance position is controlled in feed-back.

\subsection{Center of mass positioning}

One of the key conditions to not reintroduce seismic noise is minimizing the coupling among ground acceleration along arm direction and arm's tilt. As it is known [18] a ground acceleration $\ddot{z}$ generates a torque $\tau$ on the arm equal to:

$$
\tau=m \delta \ddot{z}
$$

where $m=1.75 \mathrm{~kg}$ is the arm's mass and $\delta$ is the distance from center of mass to rotation axis. To minimize this noise it is necessary to set $\delta$ within few microns: in our estimations of the final Archimedes noise of fig. 4 we choose a conservative distance $\delta=10 \mu \mathrm{m}$. Several tests have been performed to uncertain the difficulties of setting this distance within this limit. The tuning is made by regulating a set of screws placed on the upper surface of the arm, having a vertical course of $20 \mathrm{~mm}$ and $10 \mathrm{~mm}$. The sensitivity is about 1 micron for the finest screw rotation. The tuning is performed in air, then the system is evacuated and the distance measured by measuring the resonance frequency. As estimated by simulation the stiffness of the joint contributes to the frequency for about $f_{k}=3.2 \mathrm{mHz}$. The contribution of the gravitational stiffness is given by: $f_{g}=\sqrt{m g \delta} I$ where $g$ is the gravitational acceleration and $I=0.042 \mathrm{~kg} \mathrm{~m}^{2}$ is the moment of inertia of the arm. The resonance frequency is thus $f_{R}=\sqrt{f_{k}^{2}+f_{g}^{2}}$ and for $\delta=10 \mu \mathrm{m}$ the frequency $f_{R}=10.4 \mathrm{mHz}$.

The setting of the working point described above is indeed quite robust and reproducible. Settings within few microns are possible. It was in any case decided to work within the tens of microns of distances because the system shows better stability for measurements lasting several days.

\subsection{The control system}

The photodiode signal is acquired with sampling rate of $1 \mathrm{kHz}$. The filter is a usual low pass filter with couples of (real) zeroes before the resonance to properly rotate the phase, real poles after the resonance to recover the slope and further complex zeroes and poles to compensate for high frequency resonances. The actuation is made by electrostatic actuators composed by aluminum plates placed on the sides of the arm. The open-loop unity gain is at $0.2 \mathrm{~Hz}$.

\section{Results}

The main results are reported in fig. 4. The experimental sensitivity is compared with the expected signal and the expected sensitivity of the final Archimedes experiment. For frequencies above $0.1 \mathrm{~Hz}$ the sensitivity is limited by the sensing noise. For lower frequency the sensitivity is limited by the seismic noise. Indeed the ground noise present in the laboratory is quite high, 
determined both by seismic noise and anthropic noise. Good sensitivities are reached only during the night, particularly within the hours from 2 am to $4 \mathrm{am}$.

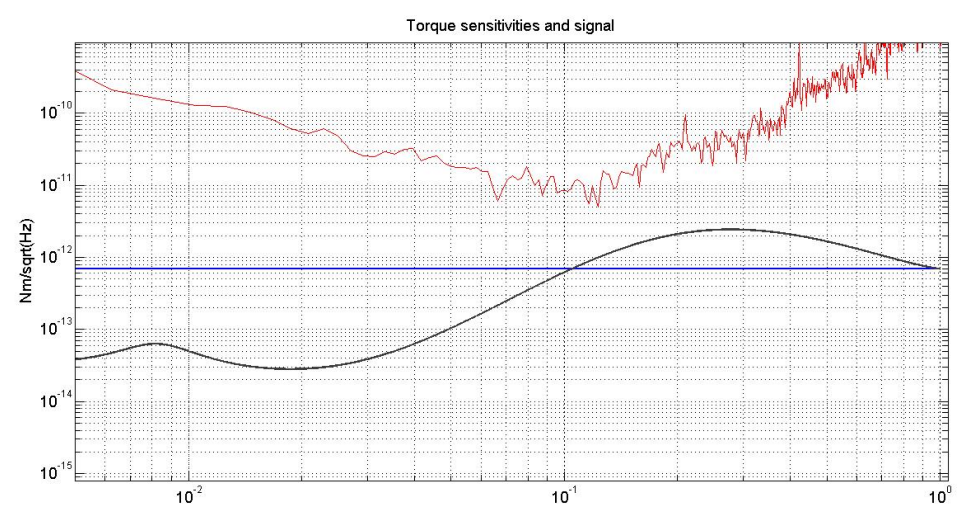

Figure 4: Prototype sensitivity (upper curve) compared with expected signal (blue line) and final sensitivity (lower curve).

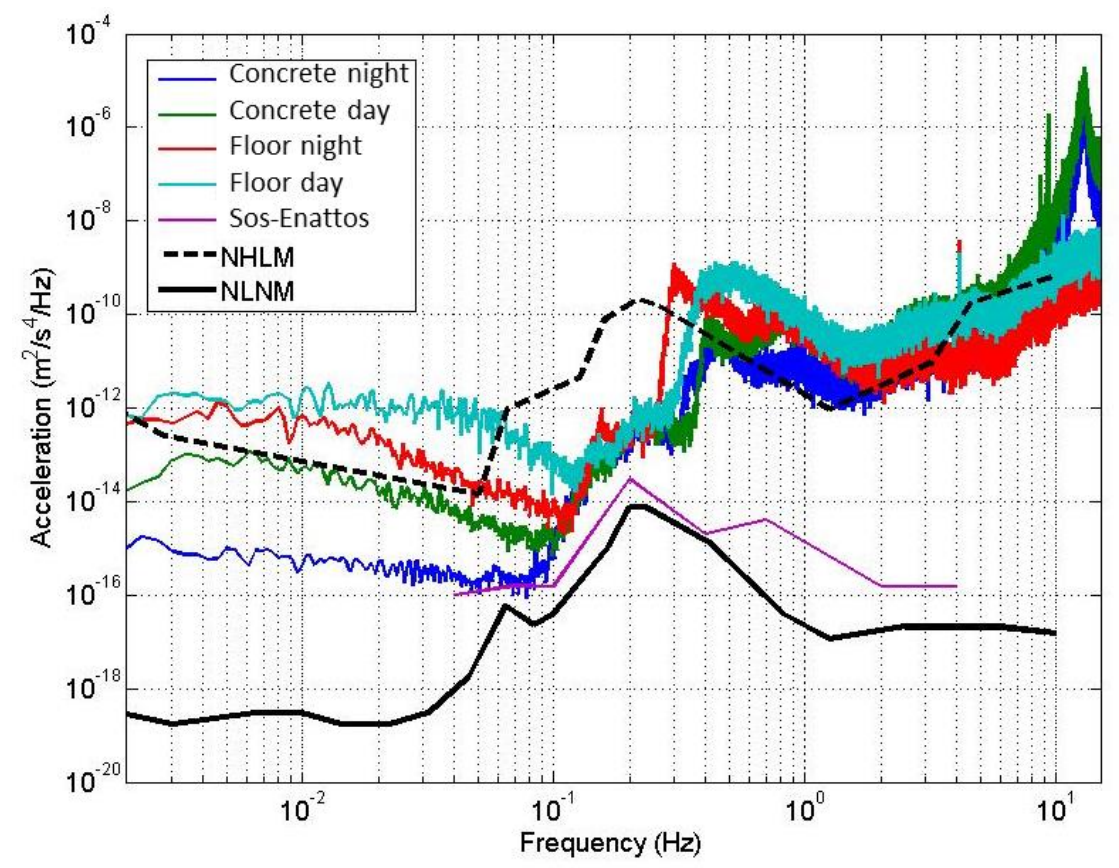

Figure 5: Seismic noise in our lab during day (cyan) and night (red), in a concrete plinth near our lab (green, blue), and in the Sos-Enattos mine (violet), compared with High and Low Peterson model [6].

The present results, in the region of 0.1 to about $2 \mathrm{~Hz}$, represents at our knowledge the best torque sensitivity in the world. The long term stability has been checked and no effects have been appreciated. The feed-back remains stable over weeks of data taking, the transfer function and operating point don't get significantly affected by lab-environment variations of temperature or humidity. All mechanical parts, particularly the joints, don't show significant aging effects on the 
time-scale of the year, even when exposed in air. In the region of lower frequencies, of tens of $\mathrm{mHz}$ and lower, the seismic noise is clearly dominating. This region is of particular interest for the final experiment and it is deserving a strong attention. At the present a low seismic and low anthropic noise underground laboratory, named $S A R-G R A V$, is under construction in the SOS-Enattos mine (Sardinia, Italy).

The laboratory seismic noise has been measured with a Trillium accelerometer and reported, for a quiet night, in fig. 5. In the figure is reported also the seismic noise as measured in SOS-Enattos site. The noise in the lab is more than four order of magnitude (in power spectral density) with respect to quiet site in almost all frequencies from few $m \mathrm{~Hz}$ to few $\mathrm{Hz}$. This conditions is important because it is yet not proved that there no noise down-converted in our system. SOS-Enattos mine is one of the lowest seismic noise site in Europe and indeed the prototype balance is expected to move in this quieter place as soon as the laboratory will be ready.

\section{Conclusion}

The balance prototype has shown that there no show-stopper in realizing the final apparatus for the measurement of the Archimedes force of vacuum. Tests on joints and balance behavior have driven the final design toward long arm and soft joints and the final project can now start. Other control tests, like robustness against thermal drifts and long term stability haven't shown showstopper problems as well. The torque sensitivity reached is the best in the intermediate frequency region and is limited in lower frequency by the high seismic noise of our laboratory. Moving in the quiet site of SOS-Enattos mine is the next step to ultimate prototype sensitivity tests and help designing the last details of final experiment.

\section{References}

[1] For an historical review see for example: H. S. Kragh, J. Overduin, âĂIJThe Weight of the Vacuum: A Scientific History of Dark EnergyâĂİ . Springer Heidelberg New York Dontrect London (2014)

[2] S. Weinberg, "The cosmological constant problem", Rev. Mod. Phys. 61, 1 (1989).

[3] T. Padmanabhan, "Why Does Gravity Ignore the Vacuum Energy?", Int. J. Mod. Phys. D 15, 2029 (2006)

[4] G. Bressi, G. Carugno, R. Onofrio, G. Ruoso: "Measurement of the Casimir force between parallel metallic surfaces", Phys.Rev.Lett. 88 (2002) 041804

[5] For a recent review: G.L. Klimchitskaya, U. Mohideen, V.M. Mostepanenko, "'The Casimir force between real materials: Experiment and theory"', Rev.Mod.Phys. 81 (2009) 1827-1885

[6] Peterson, J., 1993. "Observations and modelling of background seismic noise.” Open-file report 93-322, U. S. Geological Survey, Albuquerque, New Mexico.

[7] E. Calloni, M. De Laurentis, R. De Rosa, L. Di Fiore, G. Esposito, F. Garufi, L. Rosa, C. Rovelli, P. Ruggi, F. Tafuri: "Towards weighing the condensation energy to ascertain the Archimedes force of vacuum" Phys. Rev. D 90, 022002 (2014)

[8] A. Kempf " On the Casimir effect in the high-Tc cuprates" Journal of Physics A: Mathematical and Theoretical, Volume 41, Number 16 
[9] L. Rosa, S. Avino, E. Calloni, S. Caprara, M. De Laurentis, R. De Rosa, Giampiero Esposito, M. Grilli, E. Majorana, G. P. Pepe, S. Petrarca, P. Puppo, P. Rapagnani, F. Ricci, C. Rovelli,P. Ruggi, N. L. Saini, C. Stornaiolo, F. Tafuri: " Casimir energy for two and three superconducting coupled cavities: numerical calculations " Submitted to

[10] E. Calloni, L. Di Fiore, G. Esposito, L. Milano, and L. Rosa, "Vacuum fluctuation force on a rigid Casimir cavity in a gravitational field" Phys. Lett. A 297, 328 (2002).

[11] S. A. Fulling, K. A. Milton, P. Parashar, A. Romeo, K. V. Shajesh, and J. Wagner, " How Does Casimir Energy Fall? "Phys. Rev. D 76, 025004 (2007)

[12] G. Bimonte, E. Calloni, G. Esposito, and L. Rosa, "Energy-momentum tensor for a Casimir apparatus in a weak gravitational field" Phys. Rev. D 74, 085011 (2006); erratum Phys. Rev. D 75, 049904 (2007); erratum Phys. Rev. D 75, 089901 (2007), erratum Phys. Rev. D 77, 109903 (2008).

[13] G. Bimonte, E. Calloni, G. Esposito, L. Milano, and L. Rosa: "Towards measuring variations of Casimir energy by a superconducting cavity" Phys. Rev. Lett. 94, 180402 (2005).

[14] G. Bimonte, E. Calloni, G. Esposito, and L. Rosa: "Variations of Casimir energy from a superconducting transition" Nucl. Phys. B 726, 441 (2005). Articolo come il precedente con tutti i dettagli di calcolo e dipendenza dai parametri fisici.

[15] G. Bimonte, D. Born, E. Calloni, G. Esposito, U. Huebner, E. Il'ichev, L. Rosa, F. Tafuri, and R. Vaglio: "Low noise cryogenic system for the measurement of the Casimir energy in rigid cavities " $\mathrm{J}$. Phys. A 41, 164023 (2008).

[16] A. Allocca, G. Bimonte, D. Born, E. Calloni, G. Esposito, U. Huebner, E. Il'ichev, L. Rosa, and F. Tafuri: "Results of measuring the influence of Casimir energy on superconducting phase transitions" Jour. Super. and Novel Mag. 25, 2557 (2012).

[17] ET collaboration, âĂIJEinstein Gravitational wave Telescope Conceptual DesignâĂİ, European Commission FP7 Grant Agreement 211743

[18] K. Venkateswara, C. A. Hagedorn, M. D. Turner, T. Arp, J. H. Gundlach. Jan 16, 2014 âĂIJA high-precision mechanical absolute-rotation sensorâĂİ Rev.Sci.Instrum. 85 (2014) 015005 